\title{
The Research and Design of the Application Do- main Building Based on GridGIS
}

\author{
Zhong XIE ${ }^{1}$, Lina MA ${ }^{1,2}$, Liang WU ${ }^{1}$, Zhanlong CHEN ${ }^{1,2}$ \\ ${ }^{1}$ Faculty of Information Engineering, China University of Geosciences, Wuhan, China \\ ${ }^{2}$ Information Technology Institute, Beijing Union University, Beijing, China \\ Email:wuliang133@189.com
}

\begin{abstract}
According to the characteristic of Grid geographical information system (GridGIS), data distribution, heterogeneity and diversity in the grid environment, combining the high demand for shared resources and the purpose to realize unified and efficient management and sharing of distributed and mass resources, this paper proposed a grid GIS application building program on a number of peer-to-peer global Managers designs. This paper discussed the building process of the application domain, the selection criteria of application domain manager, the database design of application domain, and every service function module design of application domain. The integration of the application domain builds the three-tier management system of the node, the application domain, and the global management. The spatial query tests of distributed environment have shown that, the application domain is established to manage and share the distributed resources efficiently, and enhance the business processing functions in the grid environment.
\end{abstract}

Keywords: GridGIS, Grid, global manager, application domain, application domain manager

\section{Introduction}

GridGIS, combining with GIS, is the trend of grid development and grid computing technology, GridGIS integrates the geographically distributed and heterogeneous systems of various spatial data server computers, largescale retrieval storage systems, geographic information systems, virtual reality systems and so on. By high-speed internet connection, it forms the virtual super-processing environment of spatial resources information which is transparent to the user [1]. With the continuous development and improvement of GridGIS, we must deal with a wider range of resources, and resources will become increasingly distributed and heterogeneous. How to congregate the mass distribution of the resources, carry out efficient and unified management and share application are what we needs to consider.

There is not an ideal model for the grid distributed resource management. The centralized resources management in which system directory is only stored in a central node [2], inevitably can’t work well and the node is low

\footnotetext{
Supported projects: 1,the National High Technology Research and Development Program (863 program) major project "Grid GIS software and its major application"-software development of GridGIS(number: 2007AA120503); 2, The Project was Supported by the Special Fund for Basic Scientific Research of Central Colleges, China University of Geosciences(Wuhan)(number: CUGL090251); 3, Programs national natural science foundation of China(number: 40771165).
}

self-governing. A number of global managers used in this article solved performance bottleneck of the centralized management and improved the system stability.

The author of this paper discussed the application domain from the following aspects: the program to build the application domain, the application domain database ${ }^{1}$ design, and every service function module design of application domain. We build the application domain which discovers resource and meets the high demand of distributed business applications, and achieves efficient management and shares the resources in GridGIS system.

\section{The Building Program of the Application Domain and the Selection of Application Domain Manager}

\subsection{The Grid-Related Definition Set}

According to the standard definition of the grid in conjunction with study design of this paper, we give some definitions of terms for this grid resource management and sharing application:

Node: the computer which deploys middleware of GridGIS and provides the local grid services.

Grid System: the collection of all available nodes.

Global Manager: a group of grid nodes provides global grid directory service and the application domain management with the function of simultaneous update features. It is also the virtual organization. 
Application domain: Based on application requirements, the application will contain the resource implications of nodes, and integrates the nodes to form the application domain.

Application domain manager: a node in an application domain which manages information of this domain, maintains data synchronization with the global manager, and provides customer with management and applications, in order to realize the specific functions of application domain.

\subsection{Resource Managing Program Introduction in the Gridgis Environment}

The GridGIS system of this paper uses several peer to peer Global Managers and several Application domain managers. The system contains multiple peer to peer such global managers, and every global manager associates with multiple application domains. As global managers are equal, the resource information update and exchange among the global managers, global manager and application domain manager ensures integrity of all information of the application domain. It realized the efficient management of large and distributed resources.

\subsection{Build the Application Domain of Gridgis and Select the Application Domain Manager}

The application domain is the finer granularity of selfVirtual Organization (VO) which is dynamically composed of grid nodes, depending on the application requirements in the grid environment. Through the grid environment, different people and organizations access a variety of data and have different application requirements. To better manage resources and provide services, GridGIS administrator must analyze the requirements, and propose common attribute, according to a specific application to construct the application domain from the information in Global Manager.

GridGIS administrator inputs conditions of application domain constructing, global manager queries all nodes information which stored in itself, and then sends back the qualified nodes to the global administrator. The global administrator selects appropriate number of nodes according to the node information, service information, spatial data directory information, as well as specific application. At the same time the GridGIS system automatically specify a node from the selected nodes for the application domain manager, and then the construction of an application domain is completed.

At the time of the application domain construction, the information related the application domain is stored in the global manager, and the global manager maps the information to corresponding application domain manager. So, from the global manager, this application domain gets information table of the nodes, the application domain services table, the application domain resources directory table, the application domain roles table and the application domain permission table.

In fact, application domain manager should be selected in accordance with optimal principles. And this paper adopts such principles: the best machine performance of all nodes in this application domain, shortest path, and the highest score for the quality of service. Based on all the above principles, application domain manager will automatically select a node as the application domain manager.

As shown in Figure 1, The functions of the application domain manager can be mainly divided into: nodes information management of the application domain, security management of the application domain, and spatial processing operations and life-cycle management.

Node information management mainly contains: add, delete, search and change the node information. Application domain security management mainly contains control and manages the roles and permissions. The maps processing function of application domain manager is mainly to provide a number of map operation services, such as space for display, query and analysis.

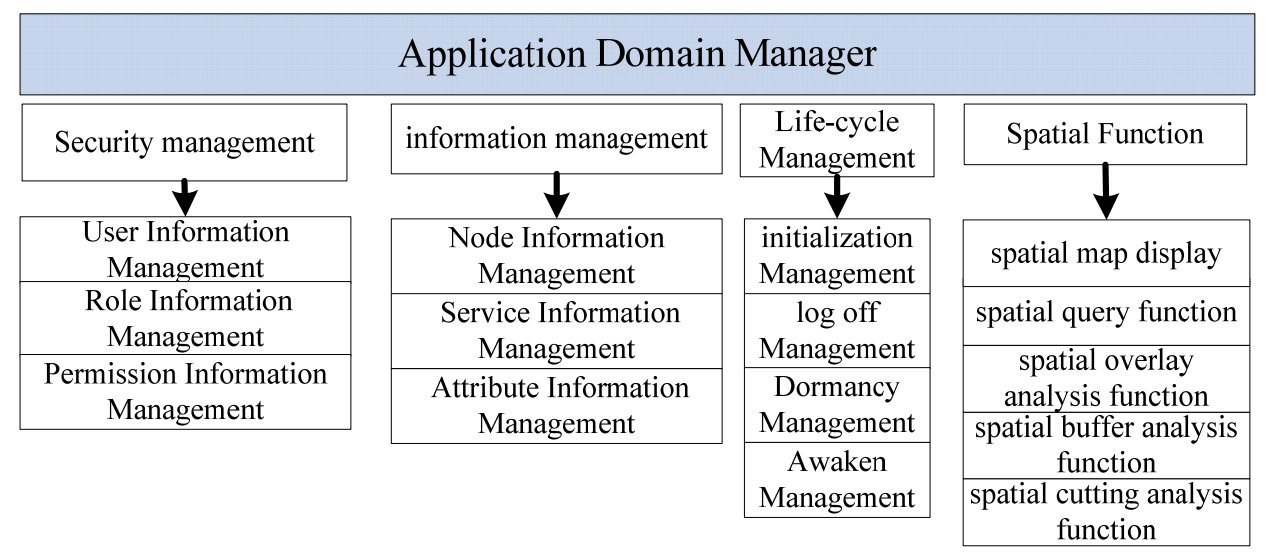

Figure 1. Modules introduction of the application domain Manage 


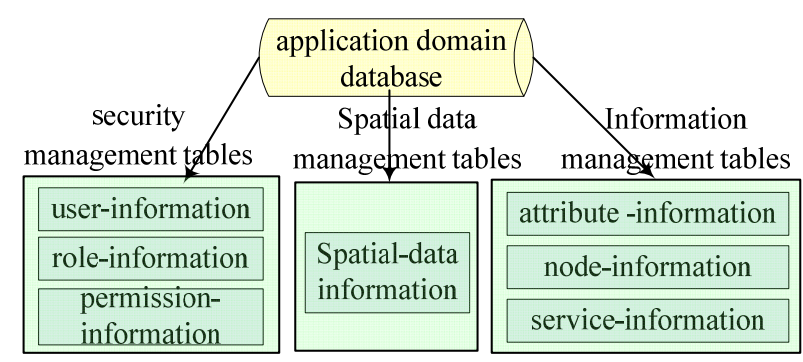

Figure 2. the tables design of application domain database

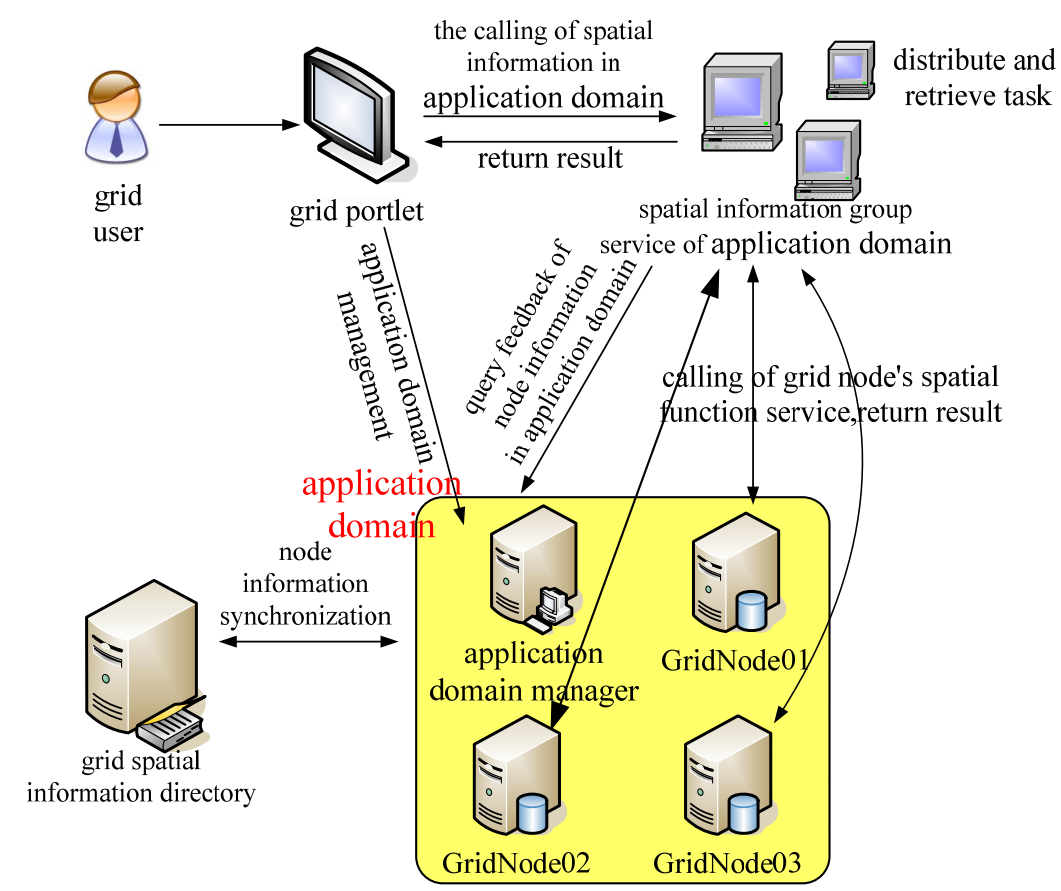

Figure 3. the entire call flow figure of the application domain services

\section{The Application Domain Database Design}

Application Domain Manager uses Oracle 9i database to store all the information of the application domain. The nodes where each application domain manager located all deploy a database. Tables in a database are mainlyconsists of three categories: security management data tables, application domain information management data tables and spatial functional data tables. As shown in Figure 2.

Security management data tables are used to store the information of users, roles, permissions, and mapping relations between the roles and the permissions. The corresponding data tables are D_UserInfo(user information tables), D_RoleInfo(role information tables), D_ PermissionInfo (permission information tables), and D_RPMap(mapping relation tables between the roles and the permissions).
Information management data tables of application domain are used to store their own attribute information related to the application domain, the provided service information, and the node information managed by the application domain. The corresponding data tables are D_Domain(attribute information of application domain tables), D_ServiceInfo(service information of application domain tables), and D_Node(node information of application domain tables).

Spatial functional data tables are used to store the spatial data information of the nodes managed by the application domain. The corresponding data table is DomainSpatialMetadata (spatial data information tables).

\section{The Application Domain Service Function Design}

The entire call flow chart of the application domain services is shown in Figure 3: 


\subsection{Security Management Service of Application Domain}

This Service provides security management function related to the application domain, which divides into user information management, role information management, permission information management and rolepermission mapping relationship management.

\subsubsection{User Information Management Service}

User information management has two parts, one is that the user manages their own information, such as registration and personal information changes. The other is the administrator manages user information, such as delete and modify user information.

User needs to register first, and after the successful registration, some of the information can be modified. Figure 4 shows the scheme of user registration and management. During the registration, through the portal cli- ent, the user logs on to the registration page, by filling out the registration information, sends a request to the global manager which accepts the request and judges the information the user filled out, if the validation is went through then sends update information request to application domain manager and notifies the user that the registration is successful. Otherwise, refuses user to register, sends failed registration message.

Administrator manages user information service which mainly provides modify and delete user information When a new user register, the administrator assign roles to user. Administrator also has the right to delete users. The process of user information management is shown in Figure 5.

When a new user registration is successful, the application domain administrator calls the user information management service to set the user's role information and other information, and assign user specific roles.

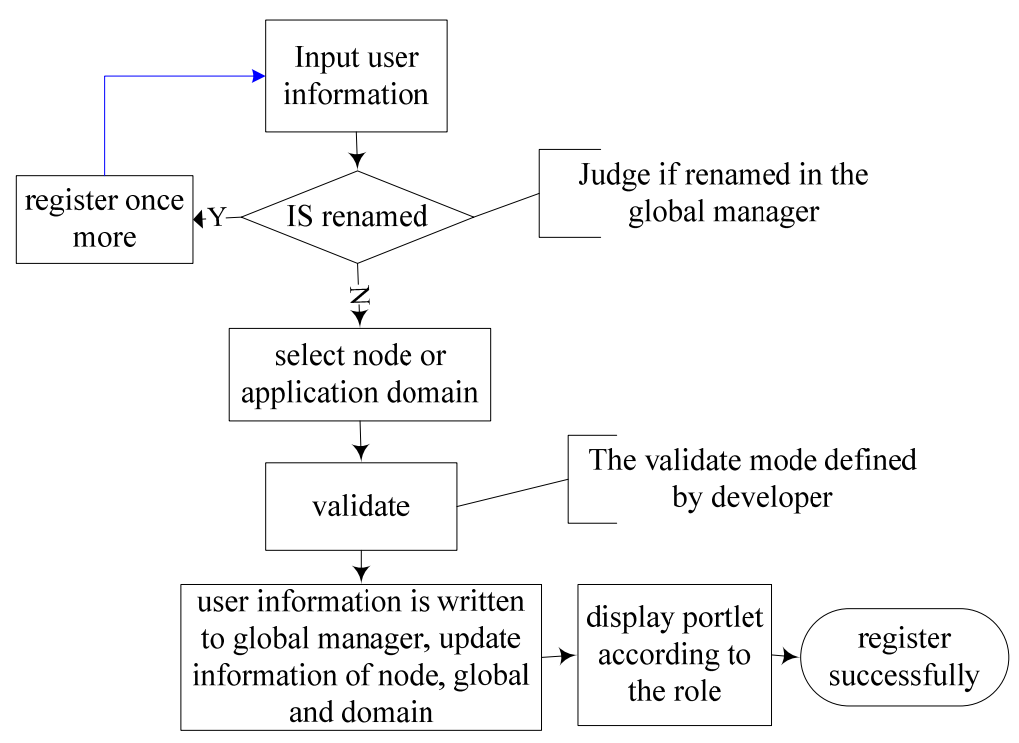

Figure 4. User Registration and Management

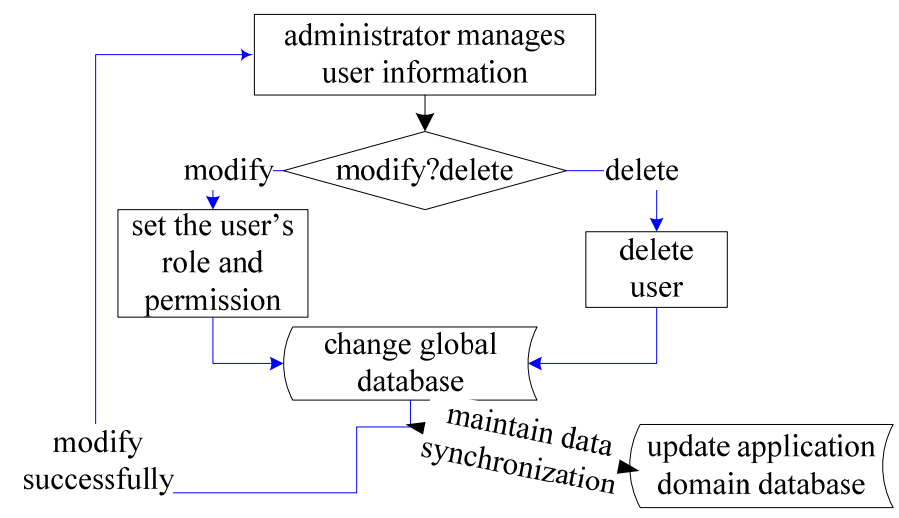

Figure 5. User Information Management 


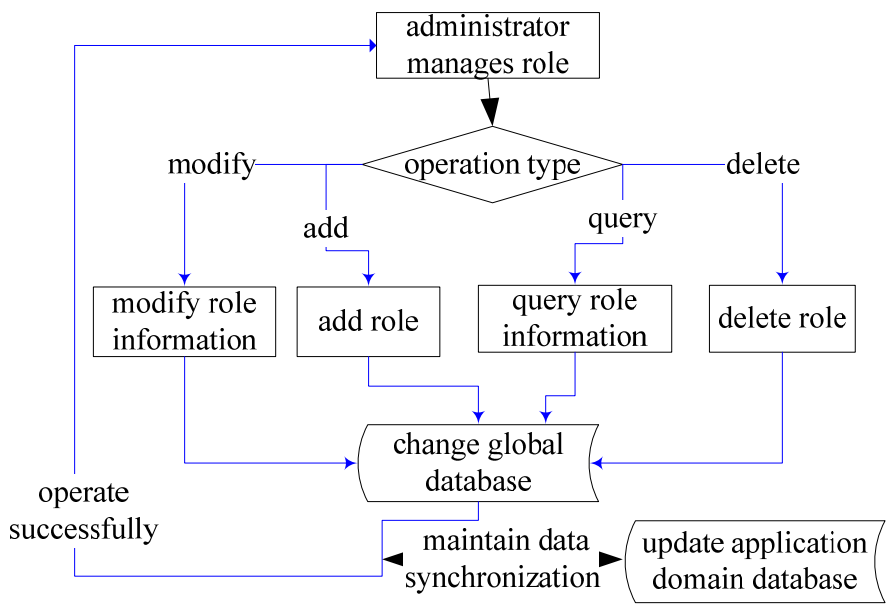

Figure 6. Role Information Management

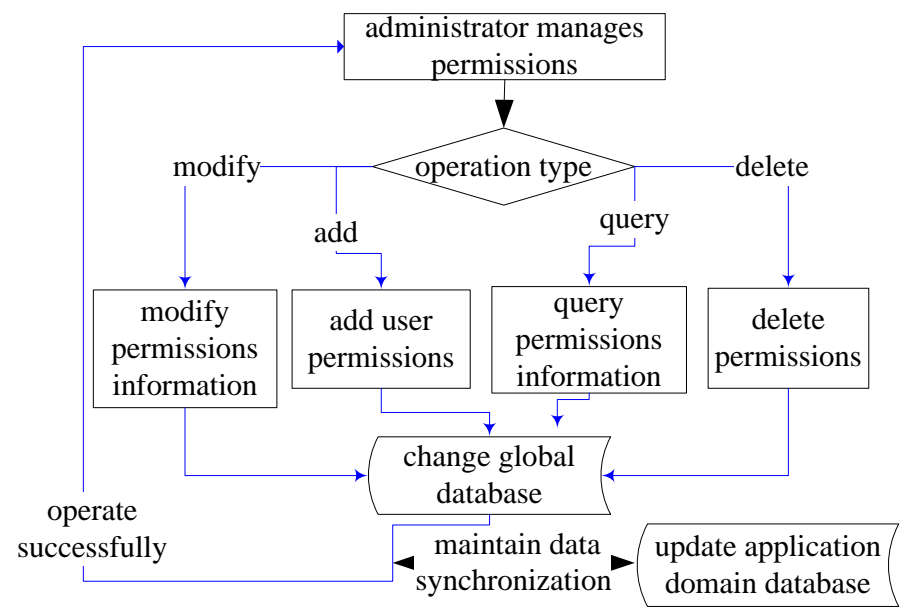

Figure 7. permissions management service

When you modify the data table of user information successfully, it will send the successful operation message to the client. Otherwise, send the failed operation message. During the running of application domain, the application domain administrator can modify specific user information according to the requirement.

After modify the user's information in application domain, it also needs to maintain data synchronization with the global manager. The application domain manager will send update request to global manager to maintain data synchronization.

\subsubsection{Role Information Management Service}

The application domain administrator designs the types of roles according to the requirement. Each role corresponds to a certain degree of Permission. The application domain administrator develops new roles, modifies and deletes the roles through the portlet managed by portalside role. Figure 6 shows the scheme of user information management.

The application domain administrator manage roles in application domain by calling roles management service, it mainly includes add, delete, query and modify role information. It will return the successful operation message after the roles database is modified successfully, otherwise return failed operation message.

It is required to maintain data synchronization with the global manager while application domain administrator modifies the role information in application domain. The application domain manager will call the synchronous updating service to the global manager to ensure the information consensus at both ends.

\subsubsection{Permissions Information Management Service} The administrator of application domain can add, modify or remove permissions according to the application requirement. Figure 7 shows the scheme of permissions management service. 
Through calling the permissions management service, the administrator can manage the permissions which mainly includes add, remove, query and modify permissions information. It returns successful operation message when the permissions of database are modified successfully, otherwise returns failed operation message.

\subsection{Application Domain Information Management Service}

The application domain node management service provides information management service of application domain, node list information and service description list services.

\subsubsection{Node Information Management Service}

The application domain manager needs to preserve the address of the node information, the spatial data information in node, and the service information in node. Application domain obtains node information from the global manager which updates the node information in application domain by calling the update service of application domain to modify the node information in the application domain manager. Application domain manages nodes information, as shown in Figure 8.

The application domain manager can delete its nodes, and when it delete a node, the information of this node will be deleted from application domain database, At the same time, the synchronous update service of global manager is called to send update request to global manager in order to ensure the consensus of node data information at both ends. Figure 9 shows the process of application domain to delete node.

\subsection{Application Domain Life-cycle Management Service}

The application domain life-cycle management functions provide functions needed by the implementation of application domain, such as create, suspend and log off application domain.

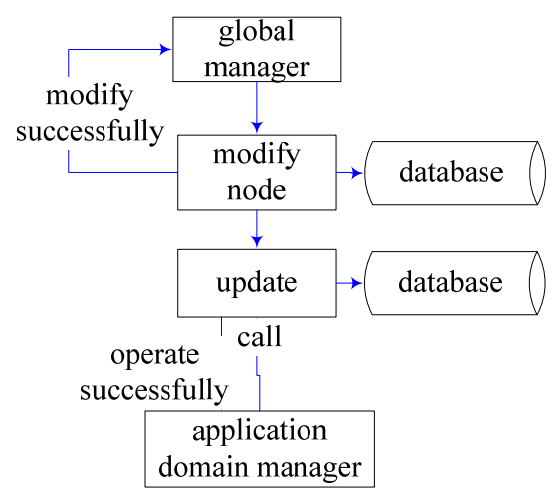

Figure 8. Application domain manages nodes informa- tion

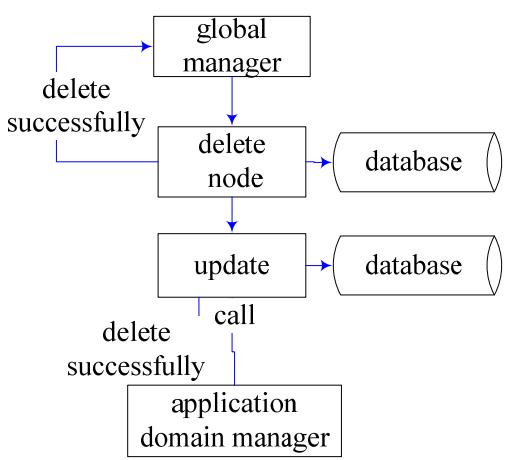

Figure 9. Application domain deletes node

The initialization functions of the application domain are used to initialize the required information when application domain is created. It mainly includes the creation of data table of application domain, getting node information and user information from global manager.

After an application domain is created, it needs to temporarily close the service on account of management demand. You can use the hibernation function, through the wake-up function to restart service.

It is required to log off service to release resources which the application domain owned when an application domain is removed or exceed the valid time, including removing all the information in data table of the application domain and stop application domain function.

\subsection{Application Domain Spatial Function Service}

The application domain spatial functions inquiry and determine the request information which is sent to the portal client. Get the node information which meets the requirements through querying the node information in database. And then sends the request to corresponding node for the actual function operation. When the node processing completed, the results will be returned to the application domain, and application domain analyses and integrates all the results. Finally returns to the portal-side to display(see Figure 10).

The application domain spatial map display function needs to calculate the scope of the map corresponded to user role in application domain firstly, that is, the scope the user can see according to corresponding permission; then the application domain will send request to nodes which can change the map to ask for a map of the designated area. After each node processing is completed, the picture will send to the application domain manager by binary stream, and the application domain manager overlay all of the pictures. Finally, the results will be returned to the portal-side to call.

The application domain spatial query function is to query a scope option through dragging a box and after selecting a certain map scope firstly; the coordinates of the scope are sent to the a application domain manager 


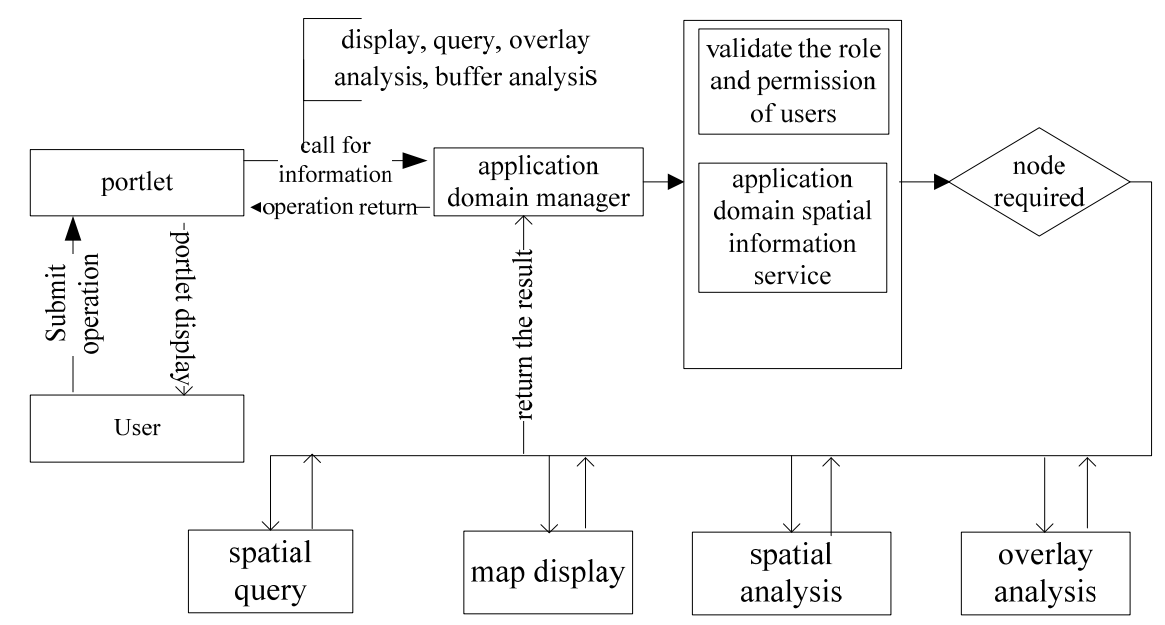

Figure 10. Application domain spatial analysis service

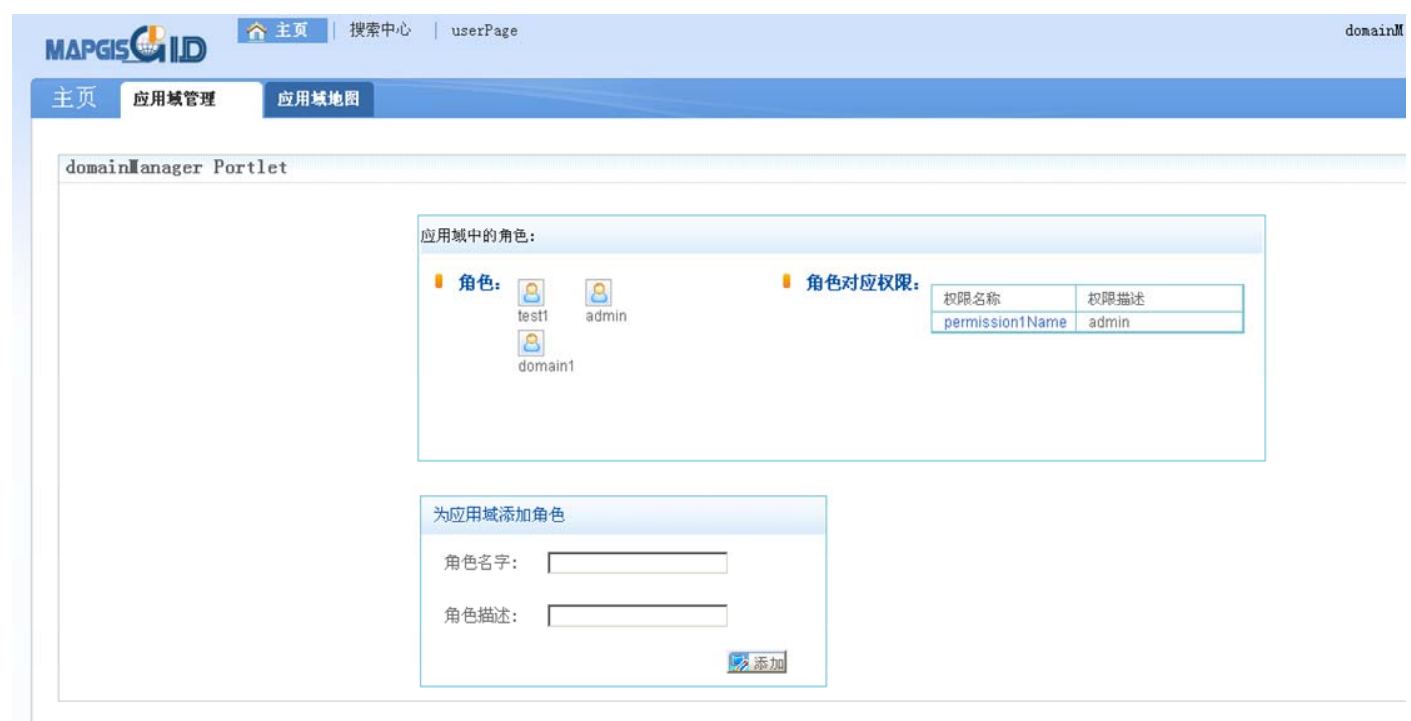

Figure 11. The management interface for application domain administrator

where the query scope will be parsed and judged in order to determine which nodes need to send request; after the request distributed is completed, the queries will be sent to the corresponding node; then the node processes the request and returns the results to application domain which will integrate the results of all nodes and return it to portal to display.

The application domain spatial overlay analysis function needs to select the overlay layer required to overlay analysis firstly; then sends the request to the application domain manager which will sent request to corresponding node according to the location of layer node; the node processes the request and returns results to application domain; the application domain manager then returns the results to portal to display.

The application domain spatial buffer analysis func- tion needs to select the layer required to be analyzed. You should set the layer to be edited firstly, and then select the region of buffer analysis, and send a request to the application domain manager. The application domain manager will parse and process the request in order to get the nodes to meet the requirement. Then send the buffer analysis request to nodes. Each node processes the request will send the ultimately results to the application domain manager which integrates the results and returns the results to portal to display.

\section{The experiment}

In our lab, we deployed eight machines to form a distributed computing environment.

It establishes a small application domain formed with four nodes in the spatial information grid support plat 

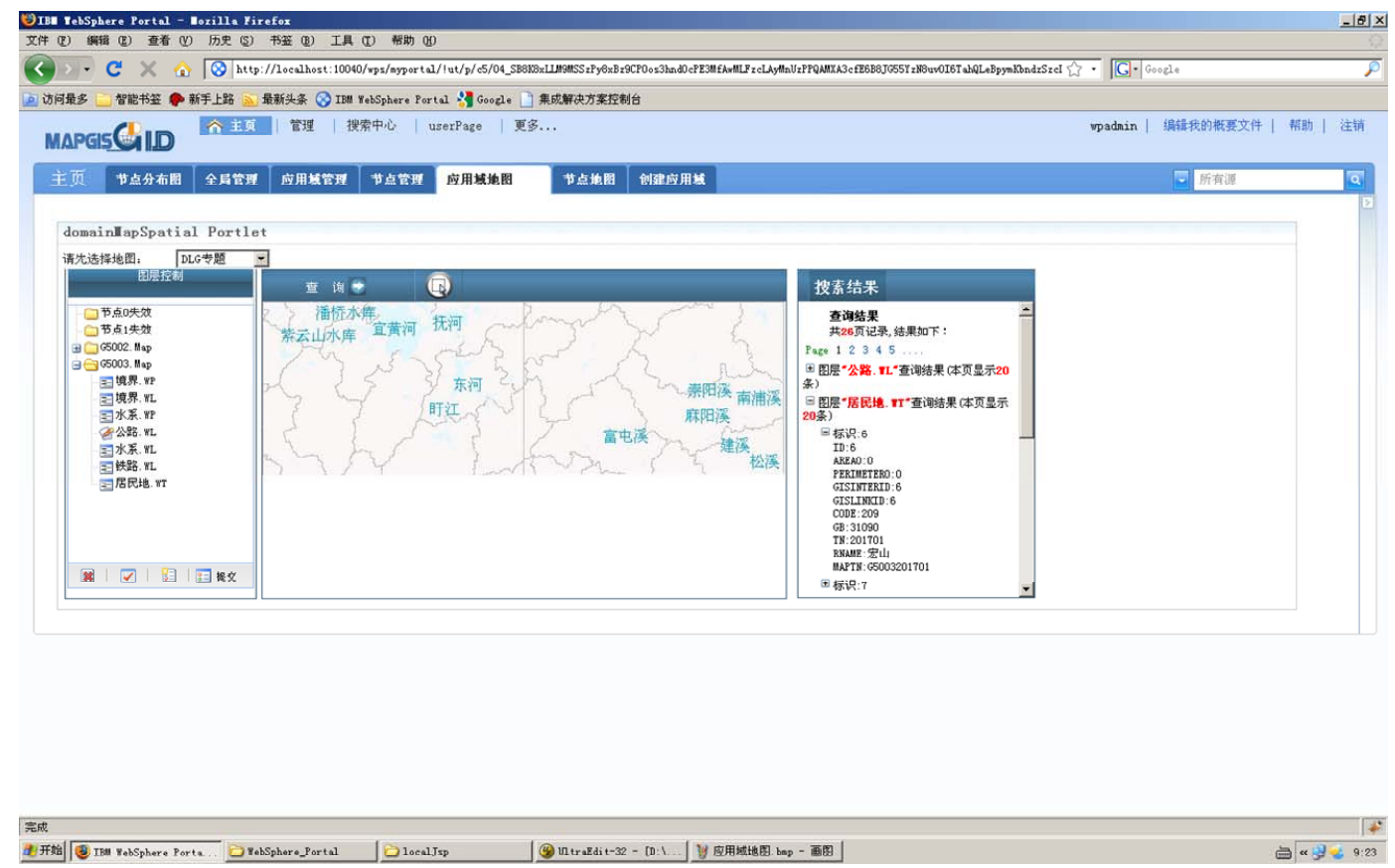

Figure 12. map query interface of application domain

form system--MapGIS Grid. Each node is installed on distributed query vector analysis software, and database is installed on the application domain manager. We carry out spatial query test for the distributed system, and get accurate and fast query results. This is the login interface for application domain administrator in figure 11. And figure 12 is query and test result figure of the application domain.

\section{Conclusion}

This article is supported by the National High Technology Research and Development Program (863 program) major project "Grid GIS software and its major application" and the project of the Special Fund for Basic Scientific Research of Central Colleges, China University of Geosciences (Wuhan).

In order to effectively manage and expediently use these large distributed data in the grid environment, we proposed a complete application domain building program based on the grid resource system management of multi-global managers. The integration of the application domain builds the three-tier management system. It makes the system nodes and global manager to play their respective advantages: the node has the greatest competence and higher self-governing, the application domain realizes the function classification and quick operation, and the global manager achieves integration and sharing of resources. The application domain man- ager shares part task of the global manager, the application domain realizes fast and easy call the distributed business, and distributed processing capabilities of GridGIS is enhanced.

\section{REFERENCES}

[1] Wang Xi, Wang Dazhong, Wang Meng. New Development of Geographical Information Technology- Preliminary Discussion of GridGIS[J]. GEOMATICS\&SPATIAL INFORMATION TECHNOLOGY, Vo.l 29, No. 4, P43-50.

[2] Tao Shiqun. A Model to Evaluate Catalog Management and Allocation Performance in Distributed Database Systems[J]. Journal of Shanxi University (Natural Science Edition), 2002,25(2):112 114.

[3] Chen Bo. The research of a peer to peer computing model and its application in grid environment [D], Institute of Software Chinese Academy of Sciences, 2004, 05

[4] Zou Rui. Research on Grid Resource Management Model[D]. master thesis, Huazhong University of Science\&Technology, 2004,05.

[5] Zhang Luwei. Design and Implementation of Distributed Directory Service in Grid Monitoring System, master thesis, Harbin Institute of Technology, 2006, 06.

[6] Wang Yuxiang. The Research of the distributed network Geographic Information System [D], Institute of Remote Sensing Applications Chinese Academy of Sciences, 2002,06. 